\title{
Global Evidence on the Rapid Adoption of Telemedicine in Primary Care During the First Year of the COVID-19 Pandemic: a Scoping Review Protocol.
}

\section{Daniela Valdes ( $\nabla$ daniela.valdes@warwick.ac.uk)}

University of Warwick Department of Computer Science https://orcid.org/0000-0002-3489-8408

\section{Lama Alqazlan}

University of Warwick Department of Computer Science

\section{Rob Procter}

University of Warwick Department of Computer Science Jeremy Dale

University of Warwick Warwick Medical School

\section{Protocol}

Keywords: Global Health, Innovation, Pandemic response, Primary care, Teleconsultation, Telemedicine

Posted Date: March 16th, 2021

DOl: https://doi.org/10.21203/rs.3.rs-302879/v1

License: (c) (1) This work is licensed under a Creative Commons Attribution 4.0 International License.

Read Full License 


\section{Abstract}

Background: Before the declaration of the COVID-19 pandemic in March 2020, primary care relied on face-to-face consultations, with relatively limited use of telemedicine. The rapid uptake that occurred following the onset of the pandemic in countries such as the United Kingdom, Canada and New Zealand prompts questions around the drivers and extent of this transformation in clinical practice at global level. The purpose of this scoping review is to explore the global evidence surrounding the rapid adoption of telemedicine in primary care settings during the first year of the COVID-19 pandemic.

Methods: A draft protocol was tested through an initial search on Ovid Medline, Web of Science and Google Scholar with additional searches on the Cochrane Database. This informed the final selection of terms which will be use to search Ovid, Web of Science, Google Scholar, PROSPERO and Cochrane Library, filtering for studies from the pandemic declaration onwards. Additional grey literature reports will be sourced through simplified searches on appropriate search engines in widely spoken languages. Duplicates will be removed by screening titles. Abstracts and grey literature text extracts will be screened based on pre-set eligibility criteria by an automated tool and further screened by the lead researcher. Abstracts (and extracts in the case of grey literature) will be mapped against the domains of the Nonadoption, Abandonment, and challenges to Scale-up, Spread and Sustainability (NASSS) framework. Data will be presented in table format.

Discussion: This review will map the current literature to identify current gaps in evidence related to the adoption of telemedicine after the declaration of the pandemic in March 2020. The use of simplified searches in the most spoken languages in the world is aimed at capturing more immediate non-academic reflections and experiences on this major service change at a global level.

Systematic review registration: Study has been registered on Open Science Framework and can be accessed through the following URL: https://osf.io/4z5ut/

\section{Background}

Pre-pandemic evidence synthesis (published/submitted in late 2019) identifies a number of 'barriers' or 'objections' that justify the low uptake of alternative consultation mediums. For instance, a rapid evidence synthesis on 'digital-first' primary care, confirmed that uptake of digital channels for patient's first point of contact was low, and identified concerns around technology, workload and confidentiality [1] as main barriers. A scoping review (focused on video consultations only) identified that this mode of delivery was not appropriate in many situations and as such face to face consultations were preferred [2].

Following the WHO's announcement on the 11 March 2020 declaring Covid-2019 a pandemic [3], the radical change seen during the first year of the pandemic indicates how previously identified barriers and objections were rapidly overcome as shown in the below examples. 
- In England, for example, data for older patients shows that the rate of remote consultations more than doubled between February and May 2020 [4], following a mandated move to total triage in the English National Health Service [5].

- In Netherlands, the shift from mainly face to face to virtual consultations happened within a week, as part of the pandemic response plans [6].

- In the United States, the implementation of the 'Expansion of telehealth with 1135 waiver' [7] signalled a critical regulatory move for Medicaid Services, with a $154 \%$ increase in the year to March 2020 [8].

A preliminary search for existing systematic reviews on this topic was conducted in PubMed and PROSPERO (an international database of prospectively registered systematic reviews in health and other areas where there is a health-related outcome [9] ) on the 23 February 2021 and 28 February 2021 respectively. Of 11 results in PubMed, only one related to primary care prescribing [10]. Of 27 protocols found in PROSPERO dealing with telemedicine since the declaration of the pandemic, only one makes direct reference to primary care [11]. Researchers with connected research were contacted given the potential overlap. The current version of the Cochrane library special collection on "Coronavirus (COVID19): remote care through telehealth" (last updated on the 6 January 2021), only includes a review on healthworkers' perceptions on telehealth in primary care [12]. Both PubMed and Prospero abstracts will be added to the screening pool.

Rationale. It is important to understand what lies at the core of the rapid shift to telemedicine following years of limited penetration. In the UK, both the Health Foundation [13] and the UK Comptroller and Auditor General [14] issued recommendations of the need of compiling lessons around service shifts accelerated by the pandemic and digital transformation respectively. This understanding is important to (i) address emerging concerns around potential disenfranchising of patients or particular population groups; (ii) support public discussions about future healthcare delivery; and (iii) evaluate and refine the change management approach based on system (and organisational) digital maturity.

To support greater understanding in these areas, this scoping review explores the move to telemedicine in the context of various health systems around the world during the first year of the pandemic, with a focus on primary care. This comparative approach around digitisation builds upon recent academic literature in the area, which so far has focused on either the digital aspect [15] or pandemic response [6], mostly in English-speaking countries. This research will seek to incorporate academic and non-academic literature through other documents in widely spoken languages to capture voices and experiences world-wide [16] .

\section{Key definitions}

Telemedicine. The review will focus on the clinical practice of telemedicine (the interaction between patient and clinician). As such it draws on the World Health Organisation's definition of telemedicine [17]. 
"The delivery of health care services, where distance is a critical factor, by all health care professionals using information and communication technologies for the exchange of valid information for diagnosis, treatment and prevention of disease and injuries [...] all in the interests of advancing the health of individuals and their communities" (World Health Organization, 2010, p9).

Telemedicine will be defined, in the negative, as those modalities of clinician/patient interaction that do not require physical presence of the individuals in the same premises (either a primary care clinic or the patient's home). In the positive, these are modalities where the clinician/patient interaction is held or mediated in a 'virtual' (as opposed to physical, premises-based) environment, through the use of a particular telecommunication technology. This definition is closely aligned with that provided by Sood et al, (2007) following a systematic review of over one hundred peer-reviewed perspectives [18]. In all cases the clinician is able to access and edit the patient's record, and the appointment might be pre-scheduled, clinician-initiated or patient-initiated, synchronous or asynchronous.

These 'virtual' environments have been further described in Olayiwola et al., (2020) [19].

- Telephone visits: conversation happens over the telephone.

- Video visits: conducted through a (secure) video platform

- E-consultations: asynchronous discussions between clinician and patient, which are initiated by the patient through a patient portal which allows to specify their complaint. In some cases, the portals include a triaging protocol and allow to capture relevant details from the patient's history.

In some settings, the terms 'econsultations' or 'econsults' can also apply to clinician/clinician discussions; but for the purposes of this review these will be excluded.

Primary care. Traditionally, primary care covers a multidisciplinary team of healthcare professionals dealing with areas related to communicable, non-communicable disease, prevention and management [20]. A standard definition is provided in the Alma Ata Declaration [21]:

"...essential health care based on practical, scientifically sound and socially acceptable methods and technology made universally accessible to individuals and families in the community through their full participation and at a cost that the community and country can afford to maintain at every stage of their development in the spirit of self-reliance and self-determination. It forms an integral part both of the country's health system, of which it is the central function and main focus, and of the overall social and economic development of the community. It is the first level of contact of individuals, the family and community with the national health system bringing health care as close as possible to where people live and work, and constitutes the first element of a continuing health care process."

The above definition also specifies this is a community-based (as opposed to a 'hospital'/secondary care service). Hospital or secondary healthcare services, as well as educational activities will be excluded from the review. Notwithstanding the multidisciplinary nature of primary care, searches will focus on the consultations of doctors and nurses as medical professionals. 
Population. The above definition of primary care also helps specify the population of interest, as healthcare services which are "universally accessible to individuals and families in the community" [21]. No population exclusion will be applied during the screening process.

\section{Methods}

The scoping review will be conducted in accordance with (i) the Joanna Briggs Institute (JBI) methodology [22], the latest update on the JBI methodological guidance [23], and the corresponding JBI evidence synthesis scoping review protocol template (Copyright @ 2014 , Aries Systems Corporation).

\section{Review questions and aims}

What is the evidence available surrounding the rapid adoption of telemedicine in primary care settings world-wide during the COVID-19 pandemic?

Specific objectives are to:

i. Understand the drivers behind the rapid adoption of telemedicine since the official declaration of Covid-19 as a pandemic in March 2020;

ii. Determine variations in the evidence related to telemedicine adoption across countries;

iii. Map the findings in the context of the NASSS framework [24] to identify gaps in evidence.

\section{Population-Concept-Context (PCC) summary}

Population. The review will focus on primary care services offered to the general population. Studies focusing on specific population groups or those suffering from particular conditions within a particular country or geographical area will be included.

Concept. The key concept under consideration is the adoption of 'telemedicine', as defined above.

Context. The context is primary care services provided during the COVID-19 pandemic in any setting or country.

\section{Design}

Table 1 below summarises the inclusion and exclusion criteria. 
Table 1

Inclusion and exclusion criteria

\begin{tabular}{|c|c|c|}
\hline Category & Inclusion & Exclusion \\
\hline \multirow[t]{2}{*}{ Population } & $\begin{array}{l}\text { primary care population or a population group/community } \\
\text { within the primary care population by their age or ethnicity } \\
\text { (such as children elderly indigenous people); or suffering } \\
\text { from a particular condition (including case studies) }\end{array}$ & \\
\hline & $\begin{array}{l}\text { Population groups defined by nature of a residential setting } \\
\text { (prison population care/nursing home population). }\end{array}$ & \\
\hline \multirow[t]{2}{*}{$\begin{array}{l}\text { Healthcare } \\
\text { setting }\end{array}$} & Primary care Practice, Primary Care, Family Care & $\begin{array}{l}\text { Other clinical settings } \\
\text { (secondary tertiary or } \\
\text { community). }\end{array}$ \\
\hline & & $\begin{array}{l}\text { Within community } \\
\text { exclude: mental health, } \\
\text { palliative care, } \\
\text { physiotherapy, dental } \\
\text { and pharmacy. }\end{array}$ \\
\hline \multirow[t]{2}{*}{ Context } & $\begin{array}{l}\text { First year of the coronavirus pandemic at global level (since } \\
\text { March 2020- March 2021). }\end{array}$ & $\begin{array}{l}\text { Telemedicine before } \\
\text { the pandemic } \\
\text { declaration. }\end{array}$ \\
\hline & & $\begin{array}{l}\text { Telemedicine but not in } \\
\text { the context of the } \\
\text { pandemic }\end{array}$ \\
\hline \multirow[t]{7}{*}{ Concept } & Telemedicine adoption or increased use. & Exclude surveys or \\
\hline & Include surveys and opinions on experiences of adoption. & $\begin{array}{l}\text { Opinions on the idea of } \\
\text { adoption. }\end{array}$ \\
\hline & $\begin{array}{l}\text { Includes systematic and other reviews as well as } \\
\text { observational studies. }\end{array}$ & $\begin{array}{l}\text { Exclude clinician to } \\
\text { clinician consultations. }\end{array}$ \\
\hline & $\begin{array}{l}\text { Include clinical trials over telemedicine systems if } \\
\text { telemedicine is replacing a face to face appointment. }\end{array}$ & \multirow{2}{*}{$\begin{array}{l}\text { Exclude other mobile } \\
\text { health apps or medical } \\
\text { apps or wider e-health } \\
\text { studies. }\end{array}$} \\
\hline & Include protocols for remote clinical assessments & \\
\hline & & $\begin{array}{l}\text { Exclude clinical trials if } \\
\text { telemedicine is not } \\
\text { used. }\end{array}$ \\
\hline & & $\begin{array}{l}\text { Excludes health } \\
\text { coaching or health } \\
\text { advisory (by non- } \\
\text { clinical volunteers.) }\end{array}$ \\
\hline
\end{tabular}

Types of sources. This scoping review will consider quantitative, qualitative and mixed methods study designs for inclusion. In addition, systematic reviews, protocols, other text and opinion papers will be considered for inclusion in the proposed scoping review. 
Search strategy. The search strategy has been developed to ensure transparency and reproducibility and the Preferred Reporting Items for Systematic Reviews and Meta-analyses for Scoping Reviews (PRISMA$\mathrm{ScR}$ [25] checklist has been used to verify its appropriateness. Search terms around telemedicine, primary care and Covid-19 have been expanded by a limited search on Ovid Medline and Web of Science, Warwick Librarian advice (which helpfully identified Covid-19 search terms from NICE [26]), and study of previous protocols in telemedicine in general practice [27] and Primary Care Cochrane Library Protocols. All identified search terms have been provided in Appendix 1. Examples of two the searches (for academic and grey literature) have been provided in Appendix 2.

Special considerations for grey literature. Given the rapid developments associated with the implementation of telemedicine in primary care settings during the first year of the pandemic, this scoping review considers emerging thinking by mapping 'grey', non-academic literature. Preference will be given to 'first-tier' grey literature, comprising of government policy and reports, as well as think-tank reports and white papers (mostly found in PDF format). This documentation is highly retrievable and credible[28]'[29]'.Similar methodology has been used in other reviews [30]' [31].

Searching for grey literature will focus on Google and Baidu (for reports in Chinese language), by selecting the first 50 results by relevance of pdf documents emerging from simplified search terms (telemedicine; "Primary Care"; Covid-19) in the top languages in the world (the top five in terms of internet users [32] and the first language of the top five countries by population [33]). Terms used for these searches were found using Machine Translation (Google Translate) and discussions with native speakers. The list of terms is found in Appendix 1.

Abstracts and excerpts for searches in other languages will focus on PDF documents published between March 2020 and the date of the search as to focus on the emerging evidence surrounding the early stages of the coronavirus pandemic period only. These will be translated using Machine Translation if no abstract in English is available [16]. In case the grey literature does not include an abstract or summary, researchers will extract three paragraphs for screening and mapping: the first full paragraph where 'telemedicine' term is found, the paragraph before and the paragraph after.

Final searches were undertaken between February and March 2021, including academic databases such as Ovid Medline, Web of Science and Google Scholar (first 50 results by relevance), as well as PROSPERO results. No language restrictions will be included in the academic searches.

\section{Evidence selection}

An initial search on Ovid Medline, Web of Science and Google Scholar was undertaken in December 2020. Two researchers with different academic backgrounds (healthcare management and computer science) independently screened all abstracts to (i) refine search terms, (ii) test the protocols' inclusion criteria, and (iii) train an automated screening tool [32]. This 'pilot' process helped refine both the search and the screening parts of the review. Note that given the 'pilot' search with academic databases to train the tool and the final searches were only undertaken months apart, there has already been independent, double- 
screening of most academic documents, and the automated tool will mostly support the screening of grey literature excerpts.

The abstracts and excerpts across all languages of the final search results will be uploaded to the automated screening tool, with suitability reviewed by the lead researcher. Reasons for exclusion of evidence that does not meet the inclusion criteria will be recorded and reported in the scoping review.

As a final part of the search strategy, the lead researcher will undertake forward and backward reference searches to identify any other potential studies that might have been missed in the search process.

\section{Data extraction}

Data will be extracted by the lead researcher from the full-text selected academic documents using an Excel template as a data extraction tool (for grey literature, the extraction will focus on the selected excerpts only). The template will include headings will such as author, title, abstract, type of document, population, concept, context, methods, key findings relevant to review questions, as well as country of study. These elements will be mapped in tabular form against the NASSS framework [24] domains on the NVIVO computer software (version 12 @ QSR International 2020). It is expected that some of the studies will touch upon one or more categories of the framework.

Any modifications to the protocol will be reported in full in the scoping review.

\section{Data analysis and presentation}

In agreement with the latest JBI methodological guidance [22] [23], no critical appraisal will be undertaken, and the final presentation of results will consist of two sections:

1. In the first section, the results of the search will be presented in a Preferred Reporting Items for Systematic Reviews and Meta-analyses for Scoping Reviews (PRISMA-ScR) flow diagram [23]

2. In the second section, the elements of the PCC inclusion criteria will be used to provide a narrative summary of the results and describe how they relate to the review objective and question. The authors will also include a mapping in tabular form against the NASSS framework [24] of the extracted data alongside basic descriptive analysis of frequency of counts of the data extracted (populations, concept, context, as well as country of origin).

\section{Discussion}

This session briefly outlines strengths and limitations of the research design.

Strengths. The JBI methodology [22] [23] for scoping reviews is recognised as appropriate in the context of the identification of literature gaps. Having a second researcher independently testing inclusion and exclusion criteria provides robustness to the training of the automated tool and can limit bias. The use of multi-language searches for grey literature is used amplify the voices of non-English-speaking countries 
and experiences. Further, using the NASSS framework [24] to map the literature is an innovative approach to the scope as it provides a tried and tested framework of study of technological transformation in healthcare.

Limitations. It is recognised that this is an emerging field in the literature and as such there is limited academic output at this stage that has been through peer review. Further review might be beneficial in a few years to capture new published academic research in this area. There are limitations in the reproducibility of google searches given that search results are regularly updated depending on the popularity of individual results over time. To mitigate known limitations in comprehensibility and usability of Machine Translation of abstracts and excerpts [35], the researchers have sought support as appropriate from native language speakers whenever feasible.

\section{Abbreviations}

- JBI - Joanna Briggs Institute

- NASSS - Non-adoption, Abandonment, and challenges to Scale-up, Spread and Sustainability

- PCC - Population, Concept, Context

- PRISMA-ScR - Preferred Reporting Items for Systematic Reviews and Meta-Analyses Extension for Scoping Reviews

\section{Declarations}

Authors' contributions. DV: Developed and drafted the protocol, undertook initial searches, discussed with librarians, screened initial results to train the tool and coordinated with co-authors. LA: Undertook screening of initial results to train the tool and provided search terms in Arabic, provided translation of research terms in Arabic and Arabic searches in google. JD provided steer throughout, provided feedback on the language used for grey literature searches and approved the search terms related to Primary Care, as well as reviewing the last draft. RP and JD provided comments to an early version of the protocol. All authors have approved the submitted version. All authors have agreed both to be personally accountable for their own contributions and to ensure that questions related to the accuracy or integrity of any part of the work, even ones in which they were not personally involved, are appropriately investigated, resolved, and the resolution documented in the literature.

Acknowledgements. The authors would like to thank Christopher Vernon and Samantha Johnson, Warwick Academic Support Librarians for their guidance at the early stages of this project. Danyang Hu, postgraduate researcher at the University of Warwick, undertook searches in Chinese on Bauidu in late December 2020. Masyithoh Annisaush Sholihah; Komal Ishtiaq; Joya Tyagi are postgraduate students at Warwick University and provided validation of the search terms in Indonesian, Urdu and Hindi respectively. 
Competing interests. At the time of submission (March 2021) DV worked as Chief Operating Officer for a healthcare organization while conducting self-funded PhD studies. The protocol has been undertaken independently from DV's primary occupation. Any findings are DV's own and do not represent her employer.

Ethics approval and consent to participate. Not applicable.

Consent for publication. Not applicable.

Availability of data and materials. Not applicable. No datasets currently available.

Funding. Not applicable.

\section{References}

1. Rodgers M, Raine G, Thomas S, Harden M, Eastwood A. Informing NHS policy in 'digital-first primary care': a rapid evidence synthesis. Health Serv Deliv Res. 2019;7:1-124.

2. Thiyagarajan A, Grant C, Griffiths F, Atherton H. Exploring patients' and clinicians' experiences of video consultations in primary care: a systematic scoping review. BJGP Open. 2020;4:bjgpopen20X101020.

3. WHO Director-General's opening remarks at the media briefing on COVID-19 - 11 March 2020 [Internet]. [cited 2021 Feb 20]. Available from: https://www.who.int/directorgeneral/speeches/detail/who-director-general-s-opening-remarks-at-the-media-briefing-on-covid-19-11 -march-2020

4. Joy M, McGagh D, Jones $N$, Liyanage $H$, Sherlock J, Parimalanathan $V$, et al. Reorganisation of primary care for older adults during COVID-19: a cross-sectional database study in the UK. Br J Gen Pract. 2020;70:e540-7.

5. Guidance and Standard operating procedures. General practice in the context of coronavirus (COVID19) Version 3.4 [Internet]. NHS England; 2020 Aug. Available from:

https://www.england.nhs.uk/coronavirus/publication/managing-coronavirus-covid-19-in-generalpractice-sop/

6. Huston P, Campbell J, Russell G, Goodyear-Smith F, Phillips RL, Weel C van, et al. COVID-19 and primary care in six countries. BJGP Open [Internet]. Royal College of General Practitioners; 2020 [cited 2020 Nov 12];4. Available from: https://bjgpopen.org/content/4/4/bjgpopen20X101128

7. Centers for Medicare \& Medicaid Services. MEDICARE TELEMEDICINE HEALTH CARE PROVIDER FACT SHEET [Internet]. 2020 [cited 2020 Nov 29]. Available from:

https://www.cms.gov/newsroom/fact-sheets/medicare-telemedicine-health-care-provider-fact-sheet

8. Koonin LM. Trends in the Use of Telehealth During the Emergence of the COVID-19 Pandemic United States, January-March 2020. MMWR Morb Mortal Wkly Rep [Internet]. 2020 [cited 2020 Dec 2];69. Available from: https://www.cdc.gov/mmwr/volumes/69/wr/mm6943a3.htm 
9. PROSPERO [Internet]. [cited 2021 Feb 28]. Available from:

https://www.crd.york.ac.uk/PROSPERO/\#aboutpage

10. Han SM, Greenfield G, Majeed A, Hayhoe B. Impact of Remote Consultations on Antibiotic Prescribing in Primary Health Care: Systematic Review. J Med Internet Res. 2020;22:e23482.

11. Haniye Sadat Sajadi MAN Seyedeh Elnaz Hosseini, Nazanin Soleimani, Azam Bazrafshani. What is the best approach to stop a COVID outbreak among healthcare staff in a primary care health center?

12. Ciara Gleeson, Dr Maureen Kelly Toby Lasserson, Robin Featherstone, Monaz Mehta Declan Devane. Coronavirus (COVID-19): remote care through telehealth. 2020 [cited 2020 Dec 23]; Available from: https://www.cochranelibrary.com/collections/doi/SC000043/full

13. Lewis R, Pereira P, Thorlby R, Warburton W. Understanding and sustaining the health care service shifts accelerated by COVID-19. 2020;17.

14. UK National Audit Office. Reportby the Comptroller and Auditor General - Digital transformation in the NHS. 2020 May p. 57.

15. Varsamis DD. Incentives and levers for digitising and integrating primary care in New Zealand, Australia and the USA. 2020;232.

16. Walpole SC. Including papers in languages other than English in systematic reviews: important, feasible, yet often omitted. J Clin Epidemiol. 2019;111:127-34.

17. World Health Organization, editor. Telemedicine: opportunities and developments in member states: report on the second Global survey on eHealth. Geneva, Switzerland: World Health Organization; 2010.

18. Sood S, Mbarika V, Jugoo S, Dookhy R, Doarn CR, Prakash N, et al. What Is Telemedicine? A Collection of 104 Peer-Reviewed Perspectives and Theoretical Underpinnings [Internet]. https://home.liebertpub.com/tmj. Mary Ann Liebert, Inc. 2 Madison Avenue Larchmont, NY 10538 USA; 2007 [cited 2021 Feb 28]. Available from: https://www.liebertpub.com/doi/abs/10.1089/tmj.2006.0073

19. Olayiwola JN, Magaña C, Harmon A, Nair S, Esposito E, Harsh C, et al. Telehealth as a Bright Spot of the COVID-19 Pandemic: Recommendations From the Virtual Frontlines ("Frontweb"). JMIR Public Health Surveill. 2020;6:e19045.

20. Beks H, Ewing G, Muir R, Charles J, Paradies Y, Clark R, et al. Mobile primary health care clinics for Indigenous populations in Australia, Canada, New Zealand and the United States: a scoping review protocol. JBI Evid Synth. 2020;18:1077-90.

21. World Health Organisation. Declaration of Alma-Ata [Internet]. 1978. Available from: https://www.euro.who.int/_data/assets/pdf_file/0009/113877/E93944.pdf

22. Aromataris E, Munn Z, editors. JBI Manual for Evidence Synthesis [Internet]. JBI; 2020 [cited 2020 Oct 15]. Available from: https://wiki.jbi.global/display/MANUAL

23. Peters MDJ, Marnie C, Tricco AC, Pollock D, Munn Z, Alexander L, et al. Updated methodological guidance for the conduct of scoping reviews. JBI Evid Synth. 2020;18:2119-26. 
24. Greenhalgh T, Wherton J, Papoutsi C, Lynch J, Hughes G, A'Court C, et al. Beyond Adoption: A New Framework for Theorizing and Evaluating Nonadoption, Abandonment, and Challenges to the ScaleUp, Spread, and Sustainability of Health and Care Technologies. J Med Internet Res. 2017;19:e367.

25. Tricco AC, Lillie E, Zarin W, O'Brien KK, Colquhoun H, Levac D, et al. PRISMA Extension for Scoping Reviews (PRISMA-ScR): Checklist and Explanation. Ann Intern Med. American College of Physicians; 2018;169:467-73.

26. NICE. Interim process and methods for developing rapid guidelines on COVID-19 [Internet]. NICE; 2020 Mar p. 12. Available from: https://www.nice.org.uk/process/pmg35

27. Downes MJ, Mervin MC, Byrnes JM, Scuffham PA. Telemedicine for general practice: a systematic review protocol. Syst Rev. 2015;4:134.

28. Adams RJ, Smart P, Huff AS. Shades of Grey: Guidelines for Working with the Grey Literature in Systematic Reviews for Management and Organizational Studies. Int J Manag Rev. 2017;19:432-54.

29. Tyndall J. How low can you go? Toward a hierarchy of Grey Literature. 2008;14.

30. Engels N, de Graav G, van der Nat P, van den Dorpel M, Bos WJ, Stiggelbout AM. Shared decisionmaking in advanced kidney disease: a scoping review protocol. BMJ Open. 2020;10:e034142.

31. Milne-Ives M, Lam C, Velthoven M van, Meinert E. The Impact of Brexit on the Pharmaceutical Supply Chain of the United Kingdom: Scoping Review Protocol. JMIR Res Protoc. 2020;9:e17684.

32. Top Ten Internet Languages in The World - Internet Statistics [Internet]. [cited 2021 Jan 17]. Available from: https://www.internetworldstats.com/stats7.htm

33. Population, total | Data [Internet]. [cited 2021 Jan 17]. Available from: https://data.worldbank.org/indicator/SP.POP.TOTL?most_recent_value_desc=true

34. Ouzzani M, Hammady H, Fedorowicz Z, Elmagarmid A. Rayyan-a web and mobile app for systematic reviews. Syst Rev. 2016;5:210.

35. Tongpoon-Patanasorn A, Griffith K. Google Translate and Translation Quality: A Case of Translating Academic Abstracts from Thai to English. PASAA J Lang Teach Learn Thail. Chulalongkorn University Language Institute; 2020;60:134-63.

\section{Supplementary Files}

This is a list of supplementary files associated with this preprint. Click to download.

- Appendix.docx

- PRISMAScRFillableChecklist1DV30.docx 